IMMUNOGENETICS

\title{
KIRs resolve the issue
}

Natural killer (NK) cells are crucial for antiviral host defence, and their activation is controlled by integrating the signals they receive through their activating and inhibitory receptors. This report, published in Science, provides the first evidence that an inhibitory NK-cell receptor - KIR2DL3 - and its ligand affect the outcome of infection with hepatitis $\mathrm{C}$ virus ( $\mathrm{HCV})$.

MHC class I molecules function as ligands for the killer-cell immunoglobulin-like receptor (KIR) family of activating and inhibitory NK-cell receptors. Certain HLA class I alleles are associated with resolution of HCV infection, so Khakoo et al. investigated whether KIRs could also influence disease outcome. The HLA-C allotypes that are ligands for the inhibitory receptors KIR2DL2 and KIR2DL3 (which are encoded by distinct alleles at a single locus) are known as HLA-C1, whereas all other allotypes (HLA-C2) are ligands for the inhibitory receptor KIR2DL1 and the activating receptor KIR2DS1. HLA-C1 homozygosity was observed at a higher frequency among individuals who resolved infection with $\mathrm{HCV}$ than among those with persistent infection. However, this effect was seen only when individuals were also homozygous for the gene encoding the HLA-C1 ligand KIR2DL3.

The association of the KIR2DL3;HLA-C1 homozygous genotype with resolution of $\mathrm{HCV}$ infection was observed for individuals receiving a small viral inoculum (through injection) but not a large inoculum, such as that provided by blood transfusion. The protective association was seen in patients from two racial groups - Caucasians and African Americans - indicating that KIR2DL3 and HLA-C1 have a synergistic and direct protective effect rather than protection being a result of linkage disequilibrium.

Although KIR2DL2 is also a ligand for HLA-C1 allotypes, KIR2DL3 homozygosity was essential for the association with HCV resolution. KIR2DL3 has a lower affinity for HLA-C1 than KIR2DL2, so the authors suggest that it is associated with protection

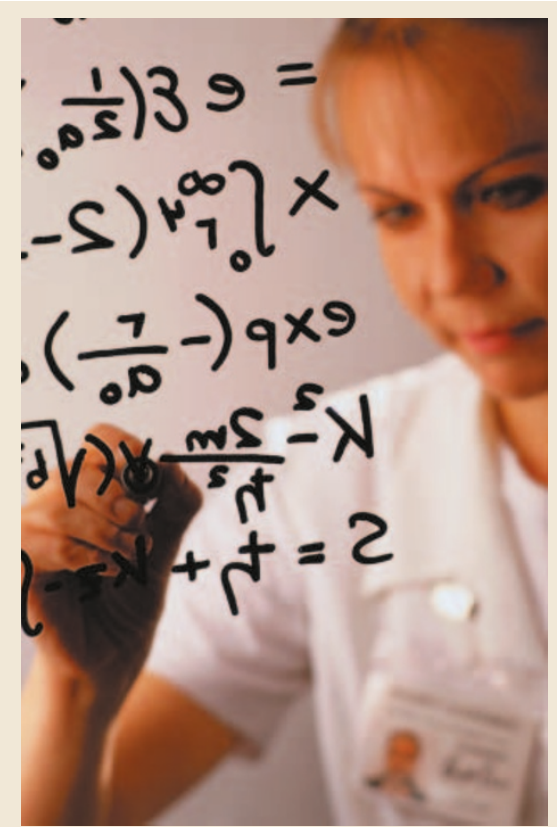

because it provides weaker inhibitory signals that allow NK cells to be more-easily activated by viral infection.

(1) References and links

Karen Honey

ORIGINAL RESEARCH PAPER Khakoo, S. I. et al.

HLA and NK cell inhibitory receptor genes in resolving

Hepatitis C virus infection. Science 305, 872-874 (2004).

\section{Separating the wheat from the chaff}
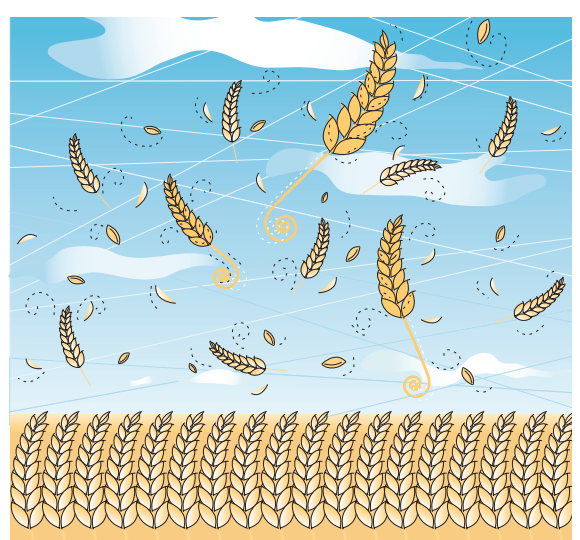

The transplantation of allogeneic haematopoietic stem cells (HSCT) - from bone marrow, peripheral blood or placental blood - is now common clinical practice after myeloablative chemotherapy or radiotherapy given for the treatment of leukaemias and lymphomas. In these patients, donor $\mathrm{CD} 8^{+} \mathrm{T}$ cells in the stemcell graft have the added benefit of mediating graft-versus-leukaemia (GVL) effects that can kill any remaining malignant cells. However, donor $\mathrm{CD}^{+} \mathrm{T}$ cells can also attack healthy tissue in the recipient, resulting in graft-versus-host disease (GVHD), which is an important cause of transplant-related mortality. A protocol that maintains the positive GVL effects of HSCT while preventing GVHD would therefore have enormous clinical benefit.

Warren Shlomchik and colleagues suggest such a protocol by showing that donor antigenpresenting cells (APCs) are required for fullstrength GVHD but not for GVL effects. They used a mouse model of bone-marrow transplantation with or without $\mathrm{CD} 8^{+} \mathrm{T}$ cells between MHC-matched but minor histocompatibility antigen-mismatched donor and recipient, as is the case for most human transplants. Mice that were transplanted with $\beta_{2}$-microglobulin-deficient $\left(\beta_{2} m^{--}\right)$bone marrow plus wild-type $\mathrm{CD} 8^{+} \mathrm{T}$ cells suffered less severe GVHD than did mice receiving wildtype bone marrow and $\mathrm{T}$ cells. Because donor APCs derived from $\beta_{2} m^{-1}$ bone marrow do not express MHC class I and therefore cannot cross-present host antigens, this indicates that donor APC function is required to maintain maximal GVHD and that donor APC depletion would reduce disease.

But what would the effect of this be on beneficial GVL responses? In a mouse model of chronic myelogenous leukaemia (CML), irradiated mice that received bone marrow plus $\mathrm{CD} 8^{+} \mathrm{T}$ cells generally survived, whereas $\mathrm{T}$-cell depletion of the transplant resulted in death from CML. The use of wild-type or $\beta_{2} m^{-/}$bone marrow did not affect the protective effect of the $\mathrm{T}$ cells, showing that donor APCs are not required for GVL killing.

Therefore, targeting donor APCs in patients with GVHD would reduce disease severity without preventing the destruction of malignant cells. Also, because APCs can quickly regenerate from donor stem cells, the immunosuppression that is associated with APC depletion would not be as long lasting as that caused by other strategies, such as T-cell depletion, that are currently used to treat GVHD.

Kirsty Minton

\section{(D) References and links}

ORIGINAL RESEARCH PAPER Matte, C. C. et al. Donor APCs are required for maximal GVHD but not for GVL. Nature Med. 1 August 2004 (doi:10.1038/nm1089).

FURTHER READING van den Brink, M. R. M. \& Burakoff, S. J. Cytolytic pathways in haematopoietic stem-cell transplantation. Nature Rev. Immunol. 2, 273-281 (2002). 